\title{
Anti-phosphatidylserine-prothrombin antibodies are associated with outcome in a TIA cohort
}

\author{
MichaelT. Mullen ${ }^{1}$, Steven R. Messé ${ }^{1}$, Scott E. Kasner ${ }^{1}$, Lauren Sansing ${ }^{2}$, M. R. Husain ${ }^{1}$, Gary L. Norman $^{3}$, \\ Zakera Shums ${ }^{3}$ and Brett L. Cucchiara ${ }^{1}$ *
}

${ }^{1}$ Department of Neurology, University of Pennsylvania, Philadelphia, PA, USA

2 Department of Neurology, University of Connecticut, Farmington, CT, USA

${ }^{3}$ INOVA Diagnostics, Inc., San Diego, CA, USA

Edited by:

Ashfaq Shuaib, University of Alberta,

Canada

Reviewed by:

Souvik Sen, University of South

Carolina School of Medicine, USA

Edward Feldmann, Brown University, USA

\section{${ }^{*}$ Correspondence:}

Michael T. Mullen and Brett L.

Cucchiara, Department of Neurology,

University of Pennsylvania, 3400

Spruce Street, 3W Gates Building,

Philadelphia, PA 19104, USA.

e-mail:michael.mullen@uphs.

upenn.edu;

cucchiar@mail.med.upenn.edu
Background: Antiphospholipid antibodies (aPLs) have been associated with thrombosis in the antiphospholipid antibody syndrome (APS) and with atherosclerotic vascular events in patients without APS. We examined the significance of aPLs in transient ischemic attack (TIA). Patients/methods: Patients with TIA $<48 \mathrm{~h}$ from symptom onset were prospectively enrolled. Traditional aPLs, including anticardiolipin and $\beta 2$-glycoprotein-I ( $\beta 2 \mathrm{GPI})$, and newer aPLs, including anti-phosphatidylserine/prothrombin (aPS/PT), $\beta 2$ GPI Domain 4/5 and $\beta 2 \mathrm{GPI}$ Domain 1 were measured. Primary outcome was a composite of stroke or death within 90 days or identification of a high risk stroke mechanism. Secondary outcomes were stroke or death and the presence of clinical/sub-clinical atherosclerosis. Results: Over 4.5 years, 167 patients were enrolled. Forty one patients (25\%) had the composite endpoint. Antibodies were measured in 158 subjects. aPS/PT IgG antibodies were significantly associated with stroke/death (OR 16.3, 95\% $\mathrm{Cl} 2.3-116.7, p=0.005)$ and were non-significantly associated with the composite endpoint (OR 4.7, 95\% $\mathrm{Cl} 0.8-29.2$, $p=0.10$ ). In multivariate analysis adjusting for $A B C D^{2}$ risk score, aPS/PT IgG remained associated with stroke/death (OR 15.7, 95\% Cl 2.0-125.6, $p=0.009$ ). Other aPLs were not associated with clinical outcome and no association between APLs and atherosclerosis was identified. Conclusion: In contrast to other aPLs, aPS/PT IgG antibodies are independently associated with stroke or death in patients with TIA.

Keywords: anticardiolipin, antiphospholipid, biomarker, infarction, thrombosis, transient ischemic attack, aPS/PT antibodies

\section{INTRODUCTION}

Antiphospholipid antibodies (aPLs) are a diverse collection of autoantibodies which are directed at plasma proteins bound to phospholipid membranes. Traditional aPLs include anticardiolipin (aCL) and $\beta$-2-glycoprotein-I ( $\beta 2 \mathrm{GPI})$ antibodies. These antibodies have been associated with thrombosis in the antiphospholipid antibody syndrome (APS), which is characterized by arterial and venous thrombosis as well as recurrent fetal loss (Cohen et al., 2010). Newly defined sub-classes of aPLs, including antibodies to $\beta 2$ GPI Domain 1 ( $\beta 2$ GPI D1) and $\beta 2$ GPI Domain $4 / 5$ ( $\beta 2$ GPI $\mathrm{D} 4 / 5)$ and anti-phosphatidylserine/prothrombin (aPS/PT), have been associated with thrombosis; although there is not yet sufficient evidence to include these antibodies into APS diagnostic criteria (Atsumi et al., 2000; Bertolaccini et al., 2005, 2011; Forastiero et al., 2005; Bizzaro et al., 2007; Sanmarco et al., 2007). Recently, aPLs have also been linked to atherosclerotic vascular disease in subjects without APS (Farsi et al., 1999; Brey et al., 2001; Staub et al., 2003; Veres et al., 2004; Iverson et al., 2006; Franck et al., 2007; Bizzaro et al., 2010). These findings raise the question of whether APL antibodies may be useful as biomarkers of vascular risk in patients without APS.
Patients with transient ischemic attack (TIA) have a high short-term risk of stroke, with estimates up to $15 \%$ at 90 days (Kleindorfer et al., 2005; Easton et al., 2009). Reliable identification of TIA patients at highest risk could be used to select patients for aggressive monitoring and therapeutic interventions. Clinical risk scores, such as the $\mathrm{ABCD}^{2}$ score, incorporate features of the patient's history and symptoms to estimate subsequent stroke risk (Johnston et al., 2007). This risk score has a number of important limitations (Cucchiara et al., 2006; Perry et al., 2011). Risk prediction may be enhanced by incorporating results of brain imaging with MRI to assess for infarction and/or vascular imaging to assess for atherosclerotic carotid stenosis, both of which are associated with a higher risk independent of the $\mathrm{ABCD}^{2}$ score (Merwick et al., 2010; Giles et al., 2011). Unfortunately, these studies are resource intensive and not always available in the emergency setting. There is a great need to identify other biomarkers which can reliably identify TIA patients who are at high risk of subsequent stroke.

Because aPLs are potentially associated with thrombosis and atherosclerosis, they may function as biomarkers of risk after TIA. The purpose of this study was to examine the association of both traditional and newer aPLs with outcome after TIA, in subjects without a clinical diagnosis of APS. 


\section{MATERIALS AND METHODS STUDY DESIGN}

This study was a retrospective analysis of a prospectively collected cohort of patients with TIA. Subjects were enrolled within $48 \mathrm{~h}$ of symptom onset. To be included subjects must have had focal neurologic deficits presumed to be due to a vascular cause. Transient neurologic symptoms which were deemed to be non-vascular by the treating neurologist were excluded. The traditional timebased definition of TIA was used (symptom resolution within $24 \mathrm{~h}$ ). Subjects were excluded if they had a terminal illness which was likely to limit their evaluation or prevent full study followup or if they were on warfarin with an INR $\geq 1.5$ at the time of enrollment. Patients on warfarin were excluded by design because of the impact of long-term anticoagulation on D-dimer, a biomarker which was previously assessed in this cohort (Cucchiara et al., 2009). For each subject, a standardized case report form was completed which captured clinical information and results of diagnostic testing. Final determination of TIA mechanism and clinical events was made at 90-day follow-up. $\mathrm{ABCD}^{2}$ clinical risk scores were calculated and categorized as low (0-3), medium (45), or high (6-7) as previously described (Johnston et al., 2007). Collection of all clinical and outcome data was blinded to results of biomarker testing. A detailed description of study methods has been previously published (Cucchiara et al., 2006). Informed consent was obtained from all subjects and the study protocol was approved by the local institutional review board.

The primary outcome measure was a composite endpoint of stroke or death within 90 days, $\geq 50 \%$ stenosis in a vessel referable to the presenting TIA, or cardioembolic source requiring anticoagulation. The endpoint was pre-specified before patient enrollment or analysis. It was designed to identify high risk TIA patients requiring emergent evaluation and specific early interventions. In observational studies, clinical interventions may prevent high risk patients from having an event. Outcomes which focus solely on clinical events may not capture these high risk patients. The combined outcome used in this study ensures that these patients are appropriately categorized. Two secondary outcome measures were: (1) stroke or death at 90 days alone, and (2) the presence of atherosclerotic disease. Atherosclerotic disease was defined to include both clinical atherosclerosis (history of coronary artery disease/myocardial infarction, stroke, peripheral vascular disease, carotid endarterectomy, or symptomatic largeartery stenosis) and sub-clinical atherosclerosis defined by the presence of atherosclerotic plaque on imaging.

\section{BLOOD SAMPLING AND ANTIBODY TESTING}

For all subjects peripheral venous blood was collected into sterile tubes containing $3.2 \%$ sodium citrate and then centrifuged at $1,300 \mathrm{~g}$ for $10 \mathrm{~min}$. Plasma was extracted and centrifuged at $10,000 \mathrm{~g}$ for $3 \mathrm{~min}$. Samples were frozen at $-80^{\circ} \mathrm{C}$ until the time of analysis. Specimens were tested for aPL antibodies at a dilution of 1:101 using the following ELISA assays: QUANTA Lite ${ }^{\circledR}$ anti- $\beta 2$ GPI IgG, IgA, IgM, anti- $\beta 2 \mathrm{GPI}$-domain $4 / 5$ IgA, anti- $\beta 2 \mathrm{GPI}$-domain $1 \mathrm{IgG}$, QUANTA Lite ${ }^{\circledR}$ anticardiolipin (ACA) IgG, IgA, IgM, QUANTA Lite $^{\circledR}$ anti-PS/PT IgG, IgM, IgG/IgM. All ELISA assays were manufactured by INOVA Diagnostics, San Diego, CA, USA and are FDAcleared for in vitro use with the exception of the $\beta 2 \mathrm{GPI}$-domain
1 IgG, $\beta 2 \mathrm{GPI}$-domain 4/5 IgA, and the PS/PT IgG/IgM kits which are research only. All tests were performed according to the manufacturer's instructions. Results for aCL and $\beta 2$ GPI were derived from five point standard curves calibrated against accepted reference standards. For all antibodies, the manufacturer's suggested thresholds were used to define a positive test. These thresholds are determined after testing clinical samples, normal controls, disease controls, and potentially cross-reactive samples.

\section{STATISTICAL ANALYSIS}

Summary statistics were computed using means, medians, and counts. Groups of patients were compared with $t$-tests, Wilcoxon ranked sum, $\chi^{2}$, and Fisher's exact test as appropriate. The primary analysis dichotomized aPL testing as positive or negative on the basis of pre-defined thresholds. We tested for an association between aPL antibodies and the composite outcome using Fisher's exact test and univariate logistic regression. Similar analyses were conducted to evaluate the relationships between aPLs and the secondary outcomes. Antibodies which were associated with outcomes with $p \leq 0.10$ were included in a multivariable model with $\mathrm{ABCD}^{2}$ score. $\mathrm{ABCD}^{2}$ score was treated as a categorical variable, with scores grouped into low, medium, and high risk (0-3, $4-5$, and 6-7, respectively). All statistical analyses were performed using STATA version 11.0 (Stata corporation, College Station, TX, USA).

\section{RESULTS}

From November 2002 to June 2007, 167 subjects were enrolled. Of these, 164 subjects were available for 90 day follow-up. Of the three subjects who were not available for 90 day follow-up, one had a carotid occlusion at presentation and was categorized as endpoint positive. The other two subjects had no events or high risk mechanisms identified at hospital discharge and were categorized as endpoint negative. For nine subjects aPLs could not be measured due to missing or insufficient blood sample volume, leaving a final sample size of 158. Baseline characteristics of enrolled subjects are summarized in Table 1.

Overall there were 40 subjects (25\%) with the composite endpoint. There were 25 subjects ( $15 \%$ ) with $>50 \%$ stenosis, $13(8 \%)$ with cardioembolism, and $8(5 \%)$ with clinical events. Of the clinical events five were strokes and three were deaths. There were five subjects who had both a clinical event and a high risk TIA mechanism. No subjects were clinically diagnosed with APS.

Using pre-defined thresholds to define positivity (Table 2), 16 of 158 subjects (10\%) were positive for traditional aPLs (aCL or $\beta 2 \mathrm{GPI}$ IgG/M). There were 19 (12\%) positive for aPS/PT IgM or IgG, and $26(16 \%)$ for $\beta 2 \mathrm{GP} 1 \mathrm{D} 4 / 5$ IgA. No subjects were positive for B2GPI D1 IgG. In the univariate analysis there was no association between traditional aPLs (aCL IgA/G/M and $\beta 2 \mathrm{GPI}$ IgA/G/M), newer $\beta 2 \mathrm{GPI}$ sub-classes (D 4/5 IgA and D1 IgG), or aPS/PT IgM with either the composite endpoint or the clinical endpoint of stroke or death. Elevated aPS/PT IgG antibodies were modestly associated with the composite endpoint, although this association did not reach statistical significance (OR 4.7, $95 \%$ CI $0.8-29.2, p=0.10)$. There was a significant association between aPS/PT IgG antibodies and the secondary endpoint of stroke or death (OR 16.3, 95\% CI 2.3-116.7, $p=0.005)$. These 
results are summarized in Table 2 . In a multivariable model (Table 3) which included $\mathrm{ABCD}^{2}$ score, aPS/PT IgG remained associated with stroke or death (OR 15.7, 95\% CI 2.0-125.6, $p=0.009$ ).

Clinical or sub-clinical atherosclerosis was present in 100 subjects $(60 \%)$. APLs were not associated with atherosclerosis (Table 2).

Table 1 | Baseline characteristics.

\begin{tabular}{lc}
\hline & $\boldsymbol{n}(\%)$ \\
\hline Age (mean \pm SD) & $62 \pm 15$ \\
Male sex & $72(46 \%)$ \\
Time from onset to enrollment, hours (mean \pm SD) & $26.4 \pm 12.7$ \\
Duration of symptoms & \\
$<10$ min & $23(15 \%)$ \\
10-59 min & $42(26 \%)$ \\
$\geq 60$ min & $93(59 \%)$ \\
Blood Pressure at Presentation & \\
Systolic (mean \pm SD) & $153 \pm 27$ \\
Diastolic (mean \pm SD) & $83 \pm 15$ \\
History of: & \\
Hypertension & $103(65 \%)$ \\
Diabetes & $34(22 \%)$ \\
CAD/Ml & $20(13 \%)$ \\
Hyperlipidemia & $63(40 \%)$ \\
Prior stroke & $28(18 \%)$ \\
PAD & $8(5 \%)$ \\
Current smoker & $26(16 \%)$ \\
Migraine & $16(10 \%)$ \\
ABCD2 Score & \\
0-3 & \\
4-5 7 & $58(37 \%)$ \\
\hline & $21(13 \%)$ \\
\hline
\end{tabular}

\section{DISCUSSION}

Patients with TIA have a high short-term risk of stroke. Biomarkers to identify patients at high risk of subsequent stroke would assist the selection of patients for aggressive monitoring and therapeutic interventions. In the cohort of subjects with TIA examined in this study, IgG antibodies against the phosphatidylserine-prothrombin complex were associated with clinical outcome (stroke or death). The association with outcome persisted after adjustment for the $\mathrm{ABCD}^{2}$ score, which is the most widely used clinical risk score for predicting outcome after TIA. In contrast, traditional aPLs (aCL and $\beta 2 \mathrm{GPI}$ ) and newer aPLs directed against the D1 and D4/5 subunits of $\beta 2$ GPI were not associated with outcome after TIA and no antibodies were associated with atherosclerosis.

Transient ischemic attack may be caused by a wide range of disease processes, including cardioembolism, large-artery atherosclerosis, non-atherosclerotic vasculopathy such as dissection, small artery lipohyalinosis, and hypercoagulable states. The pathophysiologic link between outcome after TIA and aPS/PT IgG is uncertain, but may be mediated by thrombosis, which is a common

Table 3 | Multivariable models, aPS/PT IgG positivity $>30$ units.

\begin{tabular}{|c|c|c|c|}
\hline & OR & (95\% Cl) & $p$-Value \\
\hline \multicolumn{4}{|c|}{ COMPOSITE OUTCOME } \\
\hline aPS/PT IgG & 4.3 & $(0.7-27.8)$ & 0.12 \\
\hline$A B C D^{2}$ & & & 0.07 \\
\hline $0-3$ & Ref & - & - \\
\hline $4-5$ & 1.7 & $(0.7-4.0)$ & 0.23 \\
\hline $6-7$ & 3.5 & $(1.2-10.6)$ & 0.02 \\
\hline \multicolumn{4}{|c|}{ STROKE OR DEATH } \\
\hline aPS/PT IgG & 15.7 & $(2.0-125.6)$ & 0.009 \\
\hline$A B C D^{2}$ & & & 0.15 \\
\hline $0-3$ & Ref & - & - \\
\hline $4-5$ & 2.7 & $(0.3-26.1)$ & 0.39 \\
\hline $6-7$ & 9.1 & (0.8-99.5) & 0.07 \\
\hline
\end{tabular}

Table 2 | Univariate analysis.

\begin{tabular}{|c|c|c|c|c|c|c|c|c|}
\hline & $\begin{array}{l}\text { Positive } \\
\text { cut-point }\end{array}$ & $\begin{array}{l}\text { \# Positive } \\
(n=158)\end{array}$ & $\begin{array}{l}\% \text { Positive } \\
\text { w/athero* }\end{array}$ & $p$-Value & $\begin{array}{l}\text { Composite outcome } \\
\text { OR }(95 \% \mathrm{Cl})\end{array}$ & $p$-Value & $\begin{array}{l}\text { Stroke or death } \\
\text { OR }(95 \% \mathrm{CI})\end{array}$ & $p$-Value \\
\hline aPS/PT IgG & $\geq 30$ units & $5(3 \%)$ & 100 & 0.16 & $4.7(0.8-29.2)$ & 0.10 & $16.3(2.3-116.7)$ & 0.005 \\
\hline aPS/PT IgM & $\geq 30$ units & $15(9 \%)$ & 67 & 0.79 & $1.1(0.3-3.6)$ & 0.90 & $3.5(0.6-19.2)$ & 0.15 \\
\hline $\mathrm{aCL} \lg \mathrm{G}$ & $\geq 20$ units & $3(2 \%)$ & 100 & 0.29 & $6.2(0.5-69.8)$ & 0.14 & - & - \\
\hline $\mathrm{aCL} \lg \mathrm{M}$ & $\geq 20$ units & $5(3 \%)$ & 60 & 1.00 & $0.73(0.1-6.7)$ & 0.78 & - & - \\
\hline $\mathrm{aCL} \lg \mathrm{A}$ & $\geq 20$ units & $0(0 \%)$ & - & - & - & - & - & - \\
\hline$\beta 2 \mathrm{GPI} \lg G$ & $\geq 20$ units & $2(1 \%)$ & 100 & 0.53 & - & - & - & - \\
\hline$\beta 2 \mathrm{GPI} \operatorname{lgM}$ & $\geq 20$ units & $9(6 \%)$ & 67 & 1.00 & $1.5(0.4-6.4)$ & 0.57 & $2.5(0.3-23.2)$ & 0.41 \\
\hline$\beta 2 \mathrm{GPI} \lg A$ & $\geq 20$ units & $19(12 \%)$ & 68 & 0.47 & $1.1(0.4-3.3)$ & 0.85 & $1.1(0.1-9.6)$ & 0.92 \\
\hline $\mathrm{D} 4 / 5 \lg \mathrm{A}$ & $\geq 25$ units & $26(16 \%)$ & 58 & 0.66 & $0.9(0.3-2.3)$ & 0.77 & $0.7(0.1-6.1)$ & 0.76 \\
\hline D1 lgG & $\geq 25$ units & $0(0 \%)$ & - & - & - & - & - & - \\
\hline
\end{tabular}

Legend: anti-phosphatidylserine-prothrombin (aPS/PT), $\beta$-2-glycoprotein-I ( $\beta 2$ GPI), anticardiolipin antibodies (aCL).

"Percentage of subjects who were antibody positive with clinical or sub-clinical atherosclerosis.

Blank spaces indicate no subjects who were antibody positive had the outcome of interest. 
pathologic process underlying many TIAs. The mechanism of aPLinduced thrombosis is thought to involve antibody binding to platelets and endothelial cells with subsequent activation of the clotting cascade (Horstman et al., 2009; Cohen et al., 2010). Disruption of annexin A5 anticoagulant activity has been implicated in this process, and activation of complement may also play a role (Rand, 2000; Avalos and Tsokos, 2009). aPS/PT antibodies have previously been associated with thrombosis (Bertolaccini et al., 2005). They correlate with clinical symptoms of APS and have been previously reported in subjects with acute ischemic stroke (Atsumi et al., 2000; Okuma et al., 2006). An international taskforce recently recommended a multi-center study evaluating the utility of aPS/PT as an additional marker for APS (Bertolaccini et al., 2011).

The relationship between aPLs and atherosclerosis is less well established. $\beta 2 \mathrm{GPI}$ is a component of atherosclerotic plaque (George et al., 1999). It has been hypothesized that autoantibodies to $\beta 2 \mathrm{GPI}$ contribute to the uptake of oxidized LDL by macrophages leading to the development of foam cells and plaque (Matsuura et al., 2002, 2003; Staub et al., 2006). APLs, particularly B2GPI and its $\mathrm{D} 4 / 5$ and $\mathrm{D} 1$ subunits, have been associated with clinical atherosclerotic disease (Iverson et al., 2006; Franck et al., 2007). Although atherosclerosis was common in antibody positive patients in the present study, we did not observe an association between any of the measured antibodies and atherosclerosis.

Some subsets of aPLs were relatively common in this cohort of TIA patients. For instance, IgA antibodies against $\beta 2 \mathrm{GPI}$ and the D4/5 subunit were present in a large percentage of subjects (12 and $16 \%$, respectively). In contrast, aCL antibodies were relatively uncommon, with IgG in $2 \%$, IgM in $3 \%$, and IgA in $0 \%$. This is significantly lower than the percentage of positive subjects reported in the APASS study (Anti-Phospholpid Antibodies and Subsequent thrombo-occlusive events in patients with ischemic Stroke; Levine et al., 2004). Of the 1770 subjects in APASS, $19 \%$ had aCL IgG antibodies, $5 \%$ had aCL IgM antibodies, and 10\% aCL IgA antibodies. APASS did not measure $\beta 2 \mathrm{GPI}$ or other aPLs (Levine et al., 2004). Differences in the observed prevalence of aCL may be due to differences in the study populations. APASS was nested within a clinical trial and all participants had an ischemic stroke, rather than a TIA. APASS also used a different aCL assay (Corgenix Inc., Denver, CO, USA). Differences in specificities of the assays maybe

\section{REFERENCES}

Atsumi, T., Ieko, M., Bertolaccini, M. L., Ichikawa, K., Tsutsumi, A., Matsuura, E., and Koike, T. (2000). Association of autoantibodies against the phosphatidylserineprothrombin complex with manifestations of the antiphospholipid syndrome and with the presence of lupus anticoagulant. Arthritis Rheum. 43, 1982-1993.

Audrain, M. A., Colonna, F., Morio, F., Hamidou, M. A., and Muller, J. Y. (2004). Comparison of different kits in the detection of autoantibodies to cardiolipin and beta2glycoprotein 1 . Rheumatology (Oxford) 43, 181-185. The role of complement in the antiphospholipid syndromeassociated pathology. Clin. Rev. Allergy Immunol. 36, 141-144.

Bertolaccini, M., Amengual, O., Atsumi, T., Binder, W., Laat, B. D., Forastiero, R., Kutteh, W., Lambert, M., Matsubayashi, H., Murthy, V., Petri, M., Rand, J., Sanmarco, M., Tebo, A., and Pierangeli, S. (2011). 'Noncriteria' aPL tests: report of a task force and preconference workshop at the 13th International Congress on Antiphospholipid Antibodies, Galveston, TX, USA, April 2010. Lupus 20, 191-205.
Avalos, I., and Tsokos, G. C. (2009).

partially responsible for the observed results. There is well documented variability across aPL assays. Inter-assay agreement is lower with aCL compared to $\beta 2 \mathrm{GPI}$ and lower with low-positive antibody titers, which comprised many of the positive samples in APASS (Audrain et al., 2004).

This study has several important limitations. As mentioned above, inter-assay agreement for aPLs is low. In the present study, all assays were performed with kits from the same manufacturer (INOVA Diagnostics). Our results may not be directly extrapolated to assays from other suppliers. The relatively small sample size limits power to detect associations. Although detailed clinical information is available for all subjects, the small number of clinical events limits our ability to determine whether the measured association between aPS/PT IgG and outcome is mediated by thrombosis, atherosclerosis, or some other mechanism. Furthermore, the findings should be interpreted with caution due to the small number of clinical events. There were only two patients who had both an elevated aPS/PT IgG and a clinical event. Thresholds to define positive tests were defined by the manufacturer and not specific to TIA patients. Serum was only collected at the time of the TIA. Subjects were not followed to determine if aPLs remained positive. Furthermore, a functional test of phospholipid dependent clotting was not performed as a part of the research study; although, clinically none of the subjects were suspected or diagnosed with APS. Since antibody measurements occurred after the vascular event, the possibility that aPL positivity is an epiphenomenon related to vascular occlusion or tissue injury should also be considered.

In conclusion, we found that aPS/PT IgG antibodies were associated with clinical outcome after TIA in a population of patients without APS. This association was not found with the other aPL antibodies tested. This suggests that aPS/PT IgG antibodies may be useful for risk stratifying patients after TIA; although, larger, prospective studies are needed to extend and validate these findings.

\section{ACKNOWLEDGMENTS}

Chandani McIntire, BS for technical assistance. Sources of funding: this project was supported by an American Heart Association Fellow-to-Faculty Transition Award (BC). INOVA Diagnostics providing funding for all ELISA measurements.

Bertolaccini, M. L., Atsumi, T., Koike, T., Hughes, G. R. V., and Khamashta, M. A. (2005). Antiprothrombin antibodies detected in two different assay systems. Prevalence and clinical significance in systemic lupus erythematosus. Thromb. Haemost. 93, 289-297.

Bizzaro, N., Ghirardello, A., Zampieri, S., Iaccarino, L., Tozzoli, R., Ruffatti, A., Villalta, D., Tonutti, E., and Doria, A. (2007). Anti-prothrombin antibodies predict thrombosis in patients with systemic lupus erythematosus: a 15-year longitudinal study. J. Thromb. Haemost. 5, 1158-1164.
Bizzaro, N. C. M., Villalta, D., Antico, A., Tonutti, E., and Tozzoli, R. (2010). "Antibodies to the B2GP1 domains IV/V in patients with stroke and acute coronary syndrome: results of a prospective case-control study," in Autoimmune Congress. [Abstr.]

Brey, R. L., Abbott, R. D., Curb, J. D., Sharp, D. S., Ross, G. W., Stallworth, C. L., and Kittner, S. J. (2001). beta(2)-Glycoprotein 1dependent anticardiolipin antibodies and risk of ischemic stroke and myocardial infarction: the Honolulu heart program. Stroke 32, 1701-1706. 
Cohen, D., Berger, S. P., Steup-Beekman, G. M., Bloemenkamp, K. W. M., and Bajema, I. M. (2010). Diagnosis and management of the antiphospholipid syndrome. BMJ 340, c2541c2541.

Cucchiara, B. L., Messe, S. R., Sansing, L., MacKenzie, L., Taylor, R. A., Pacelli, J., Shah, Q., Pollak, E. S., and Kasner, S. E. (2009). D-dimer, magnetic resonance imaging diffusion-weighted imaging, and ABCD2 score for transient ischemic attack risk stratification. J. Stroke Cerebrovasc. Dis. 18, 367-373.

Cucchiara, B. L., Messe, S. R., Taylor, R. A., Pacelli, J., Maus, D., Shah, Q., and Kasner, S. E. (2006). Is the ABCD score useful for risk stratification of patients with acute transient ischemic attack? Stroke 37, 1710-1714.

Easton, J. D., Saver, J. L., Albers, G. W., Alberts, M. J., Chaturvedi, S., Feldmann, E., Hatsukami, T. S., Higashida, R. T., Johnston, S. C., Kidwell, C. S., Lutsep, H. L., Miller, E., and Sacco, R. L. (2009). Definition and evaluation of transient ischemic attack: a scientific statement for healthcare professionals from the American Heart Association/American Stroke Association Stroke Council; Council on Cardiovascular Surgery and Anesthesia; Council on Cardiovascular Radiology and Intervention; Council on Cardiovascular Nursing; and the Interdisciplinary Council on Peripheral Vascular Disease. Stroke 40, 2276-2293.

Farsi, A., Domeneghetti, M. P., Fedi, S. Capanni, M., Giusti, B., Marcucci, R., Giurlani, L., Prisco, D., Passaleva, A., Gensini, G. F., and Abbate, R. (1999). High prevalence of antibeta2 glycoprotein I antibodies in patients with ischemic heart disease. Autoimmunity 30, 93-98.

Forastiero, R., Martinuzzo, M., Pombo, G., Puente, D., Rossi, A., Celebrin, L., Bonaccorso, S., and Aversa, L. (2005). A prospective study of antibodies to beta2-glycoprotein I and prothrombin, and risk of thrombosis. J. Thromb. Haemost. 3, 1231-1238.

Franck, M., Staub, H. L., Petracco, J. B., Norman, G. L., Lassen, A. J., Schiavo, N., Borges, R. B., and Von Muhlen, C. A. (2007). Autoantibodies to the atheroma component beta2-glycoprotein I and risk of symptomatic peripheral artery disease. Angiology 58, 295-302.

George, J., Harats, D., Gilburd, B., Afek, A., Levy, Y., Schneiderman, J., Barshack, I., Kopolovic, J., and Shoenfeld, Y. (1999). Immunolocalization of beta2-glycoprotein I (apolipoprotein $\mathrm{H}$ ) to human atherosclerotic plaques: potential implications for lesion progression. Circulation 99, 2227-2230.

Giles, M. F., Albers, G. W., Amarenco, P., Arsava, E. M., Asimos, A. W., Ay, H., Calvet, D., Coutts, S. B., Cucchiara, B. L., Demchuk, A. M., Johnston, S. C., Kelly, P. J., Kim, A. S., Labreuche, J., Lavallee, P. C., Mas, J. L., Merwick, A., Olivot, J. M., Purroy, F., Rosamond, W. D., Sciolla, R., and Rothwell, P. M. (2011). Early stroke risk and ABCD2 score performance in tissuevs. time-defined TIA: a multicenter study. Neurology 77, 1222-1228.

Horstman, L. L., Jy, W., Bidot, C. J., Ahn, Y. S., Kelley, R. E., Zivadinov, R., Maghzi, A. H., Etemadifar, M., Mousavi, S., and Minagar, A. (2009). Antiphospholipid antibodies: paradigm in transition. J. Neuroinflammation 6, 3 .

Iverson, G. M., Von Mühlen, C. A., Staub, H. L., Lassen, A. J., Binder, W., and Norman, G. L. (2006). Patients with atherosclerotic syndrome, negative in anti-cardiolipin assays, make IgA autoantibodies that preferentially target domain 4 of $\beta 2$-GPI. $J$. Autoimmun. 27, 266-271.

Johnston, S. C., Rothwell, P. M., Nguyen-Huynh, M. N., Giles, M. F., Elkins, J. S., Bernstein, A. L., and Sidney, S. (2007). Validation and refinement of scores to predict very early stroke risk after transient ischaemic attack. Lancet 369, 283-292.

Kleindorfer, D., Panagos, P., Pancioli, A., Khoury, J., Kissela, B., Woo, D., Schneider, A., Alwell, K., Jauch, E., Miller, R., Moomaw, C., Shukla, R., and Broderick, J. P. (2005). Incidence and short-term prognosis of transient ischemic attack in a population-based study. Stroke 36, 720-723.

Levine, S. R., Brey, R. L., Tilley, B. C., Thompson, J. L., Sacco, R. L., Sciacca, R. R., Murphy, A., Lu, Y., Costigan, T. M., Rhine, C., Levin, B. Triplett, D. A., and Mohr, J. P. (2004). Antiphospholipid antibodies and subsequent thrombo-occlusive events in patients with ischemic stroke. JAMA 291, 576-584.

Matsuura, E., Kobayashi, K., Koike, T., and Shoenfeld, Y. (2002). Autoantibody-mediated atherosclerosis. Autoimmun. Rev. 1 , 348-353.

Matsuura, E., Kobayashi, K., Koike, T., Shoenfeld, Y., Khamashta, M. A., and Hughes, G. R. (2003). Oxidized lowdensity lipoprotein as a risk factor of thrombosis in antiphospholipid syndrome. Lupus 12, 550-554.

Merwick, A., Albers, G. W., Amarenco, P., Arsava, E. M., Ay, H., Calvet, D., Coutts, S. B., Cucchiara, B. L. Demchuk, A. M., Furie, K. L., Giles, M. F., Labreuche, J., Lavallee, P. C., Mas, J. L., Olivot, J. M., Purroy, F., Rothwell, P. M., Saver, J. L., Sheehan, O. C., Stack, J. P., Walsh, C., and Kelly, P. J. (2010). Addition of brain and carotid imaging to the $\operatorname{ABCD}(2)$ score to identify patients at early risk of stroke after transient ischaemic attack: a multicentre observational study. Lancet Neurol. 9, 1060-1069.

Okuma, H., Kitagawa, Y., Kobori, S. Sekiyama, S., and Takagi, S. (2006). Prevalence rates of antiphospholipid antibodies in ischemic stroke patients. Intern. Med. 45, 1017-1018.

Perry, J. J., Sharma, M., Sivilotti, M. L., Sutherland, J., Symington, C., Worster, A., Emond, M., Stotts, G., Jin, A. Y., Oczkowski, W. J., Sahlas, D. J., Murray, H. E., MacKey, A., Verreault, S., Wells, G. A., and Stiell, I. G. (2011). Prospective validation of the ABCD2 score for patients in the emergency department with transient ischemic attack. CMAJ 183, 1137-1145.

Rand, J. H. (2000). Antiphospholipid antibody-mediated disruption of the annexin- $\mathrm{V}$ antithrombotic shield: a thrombogenic mechanism for the antiphospholipid syndrome. J. Autoimmun. 15, 107-111.

Sanmarco, M., Gayet, S., Alessi, M. C., Audrain, M., De Maistre, E., Gris, J. C., De Groot, P. G., Hachulla, E., Harle, J. R., Sie, P., and Boffa, M. C. (2007). Antiphosphatidylethanolamine antibodies are associated with an increased odds ratio for thrombosis. A multicenter study with the participation of the European Forum on antiphospholipid antibodies. Thromb. Haemost. 97, 949-954.

Staub, H. L., Franck, M., Ranzolin, A., Norman, G. L., Iverson, G. M., and Von Mühlen, C. A. (2006). IgA antibodies to beta2-glycoprotein I and atherosclerosis. Autoimmun. Rev. 6, 104-106.

Staub, H. L., Norman, G. L., Crowther, T., Da Cunha, V. R., Polanczyk, A., Bohn, J. M., Fernandes, J. G., Chahade, W. H., and Von Muhlen, C. A. (2003). Antibodies to the atherosclerotic plaque components beta2glycoprotein I and heat-shock proteins as risk factors for acute cerebral ischemia. Arq. Neuropsiquiatr. 61, 757-763.

Veres, K., Lakos, G., Kerenyi, A., Szekanecz, Z., Szegedi, G., Shoenfeld, Y., and Soltesz, P. (2004). Antiphospholipid antibodies in acute coronary syndrome. Lupus 13, 423-427.

Conflict of Interest Statement: Michael T. Mullen, MD, none; Steven R. Messé, $\mathrm{MD}$, none; Scott E. Kasner, MD, has received modest honoraria for consulting from INOVA Diagnostics; Lauren Sansing, MD, none; M. Rizwan Husain, $\mathrm{MD}$, none; Gary L. Norman, $\mathrm{PhD}$, employee of INOVA Diagnostics, Inc.; Zakera Shums, MS, employee of INOVA Diagnostics, Inc.; Brett L. Cucchiara, $\mathrm{MD}$, has received modest honoraria for consulting from INOVA Diagnostics.

Received: 15 June 2012; paper pending published: 10 July 2012; accepted: 10 September 2012; published online: 28 September 2012.

Citation: Mullen MT, Messé SR, Kasner SE, Sansing L, Husain MR, Norman GL, Shums $Z$ and Cucchiara BL (2012) Anti-phosphatidylserine-prothrombin antibodies are associated with outcome in a TIA cohort. Front. Neur. 3:137. doi: 10.3389/fneur.2012.00137

This article was submitted to Frontiers in Stroke, a specialty of Frontiers in Neurology.

Copyright (c) 2012 Mullen, Messé, Kasner, Sansing, Husain, Norman, Shums and Cucchiara. This is an open-access article distributed under the terms of the Creative Commons Attribution License, which permits use, distribution and reproduction in other forums, provided the original authors and source are credited and subject to any copyright notices concerning any third-party graphics etc. 